\title{
The X-ray Radiation Response of CdZnTe Crystal Nuclear Detector
}

\author{
X.Y. DAI, H. MENG, Y.L. ZHANG, J. TAO, R. XU, J.J. ZHANG, J. HUANG, L. WANG, \\ K. TANG, J.H. MIN, L.J. WANG* \\ School of Materials Science \& Engineering, Shanghai University, Shanghai 444, China
}

\begin{abstract}
The X-ray radiation response and radiation damage of CdZnTe (CZT) detector were studied in this paper. After rapid temperature annealing, the linearity of I-V curve was much better than before. Photo current of Au/CZT planar detector increased linearly with the X-ray intensity. An inflection point around 10$30 \mathrm{~V}$ was observed for different X-ray intensity. Below the inflection points, with the increase of applied voltage, photon current increased linearly, while the current still increased after the inflection point likely due to the illumination-related impact ionization effect. In addition, under exposure in strong X-ray intensity, the photo current remains unchanged before the inflection point while it changes significantly with after the inflection point.
\end{abstract}

KEYWOEDS: CdZnTe; radiation response; radiation damage;

\section{INTRODUCTION}

The ternary compound of cadmium zinc telluride $(\mathrm{CZT})$ is a semiconductor with properties that are well suited to the application of room temperature $\mathrm{X} /$ gamma-ray radiation detection.[2-3], [8], [11] The high atomic numbers of cadmium and tellurium ensure superior stopping power compared with silicon, gallium arsenide or germanium. Besides, the wide bandgap of CZT provides the ability to operate at room temperature whilst maintaining spectroscopic performance. For the reason that CZT detectors are widely applied in different fields in recent years. CZT bulk crystals can contain significant concentrations of defects, for example $\mathrm{Cd}$ vacancies, Te precipitates, impurities, and grains. [10] The quality and yield of CZT material has steadily increased in recent years and the limit in performance is now mainly due to the quality of the metal contacts.[1]

The performance of the metal contacts deposited on CZT must be improved to match the improvement in crystal quality. Usually $\mathrm{Au}, \mathrm{Pt}$ or $\mathrm{Pd}$ is used, which can form a quasi-ohmic contact, eliminating the problem of spectral degradation due to the polarization effect.[13] Compared with Pt or $\mathrm{Pd}, \mathrm{Au}$ is a general choice for the contact, because it can be deposited by means of several techniques and a sharp interface with little intermixing is usually obtained.[4],[12] Research on the improvements of electrode contact, with the purpose of reducing detector leakage currents, has been reported.[5-7] On the purpose of getting a better ohmic contact between the contact and the CZT bulk, rapid temperature annealing (RTA) method had been adopted.

The previous studies usually focused on the energy spectrum analysis and the improvement energy resolution. Few people have studied radiation response under X-ray with a relative low energy.

In this work we study the structural and electronic properties of $\mathrm{Au} / \mathrm{CZT}$ detector. After RTA, the linearity of $\mathrm{I}-\mathrm{V}$ curve is much better than before. Photo current is increases with the X-ray intensity. There exist an inflection point around 10-30 V for different X-ray intensity. Below the inflection points, with the increase of applied voltage, photon current increases linearly. After the inflection point, the current still increases because of the illumination-related impact ionization effect. In addition, the photon current does not change over time before the inflection point, while it changes after the inflection point under a strong X-ray radiation.

\section{EXPERIMENTAL PROCEDURE}

Single-crystal CZT samples with $10 \times 10 \times 1 \mathrm{~mm}^{3}$ in size, were lapping followed by $0.05 \mu \mathrm{m}$ alumina slurry polishing. After that, they were chemically etched with $2 \%$ bromine/methanol for $2 \mathrm{~min}$ and 
rinsed with methanol. Just before deposition, all the samples and gold wires were ultrasonically cleaned in ethanol for $10 \mathrm{~min}$. In order to minimize the surface re-oxidation, the Au contacts were deposited on the processed surfaces immediately after ultrasonically cleaning.

Samples were configured with planar contacts deposited by traditional vacuum evaporation method. Au contacts were deposited on both sides of bulk with the geometry of square $\left(7 \times 7 \mathrm{~mm}^{2}\right)$. The purity of gold wires was $99.99 \%$. Each time, accurate $3 \mathrm{~cm}$ length of gold wire was used on the Mo boat inside the vacuum evaporation equipment. Therefore, we can greatest possibly get the same thickness $\mathrm{Au}$ films.

After contact deposition, The X-ray source (30 $\mathrm{keV} 5 \sim 20 \mathrm{~mA}$ ) equipped with $\mathrm{Cu}$ target was placed directly to the center of contact and the X-ray radiation response was measured by current-voltage measurements (Keithley 2400). In order to get a better ohmic contact between the contact and the CZT bulk, samples were placed under nitrogen atmosphere in $500^{\circ} \mathrm{C}$ for 10 seconds to finish the annealing (RTA). Field emission scanning electron microscopy (FESEM, JSM-6701F) were used to analysis surface topography of Au contacts.

\section{RESULTS AND DISCUSSION}

The topography of sample contact, evaluated by scanning electron microscopy (SEM), is displayed in Figure 1. The $\mathrm{Au}$ particles can be divided into two parts. One is around $150-300 \mathrm{~nm}$, and the other is much smaller, below 100nm. This may result from the fast deposition by evaporation since the growth of Au film is finished within 3 seconds. Therefore, the deposition rate is so quick that different nucleation process happens. Additionally, it can be found in Figure 1-b that the thickness of the gold film is about $200 \mathrm{~nm}$ and gold grains are arranged very closely therefore there is nearly no pinhole or void between grains.

The dark and photo current of Au/CZT detectors before and after RTA are shown in Figure 2. Before annealing, there exists a leakage channel when the voltage is larger than $120 \mathrm{~V}$. This may arise from some voids or pinhole within the interface between $\mathrm{Au}$ and CZT. After annealing, the interface becomes uniform and this leakage channel is eliminated. The linearity of I-V curve after annealing is much better than before. The resistivity is calculated to be 2.90 $\times 10^{10} \Omega \cdot \mathrm{cm}$. The samples are tested under the same $\mathrm{X}$-ray illumination condition using a $\mathrm{Cu}$ target with a $30 \mathrm{KeV}$ and $20 \mathrm{~mA}$. The photo current of samples after annealing is one magnitude order larger than that before annealing. The loss of photo generated carriers is due to the recombination processes happening in bulk and interface. The reason for this may come from the better interface structure for the sample after annealing since the RTA process have much influenced on the interface, which may lead to eliminate the small pinhole in interface and thus decrease the recombination center of interface. Besides, for both the dark and photo I-V curves, the forward and reverse current is not completely symmetrical. This may be due to non-controllable process lead to differences between both electrodes and eventually leading to the not completely symmetrical current.
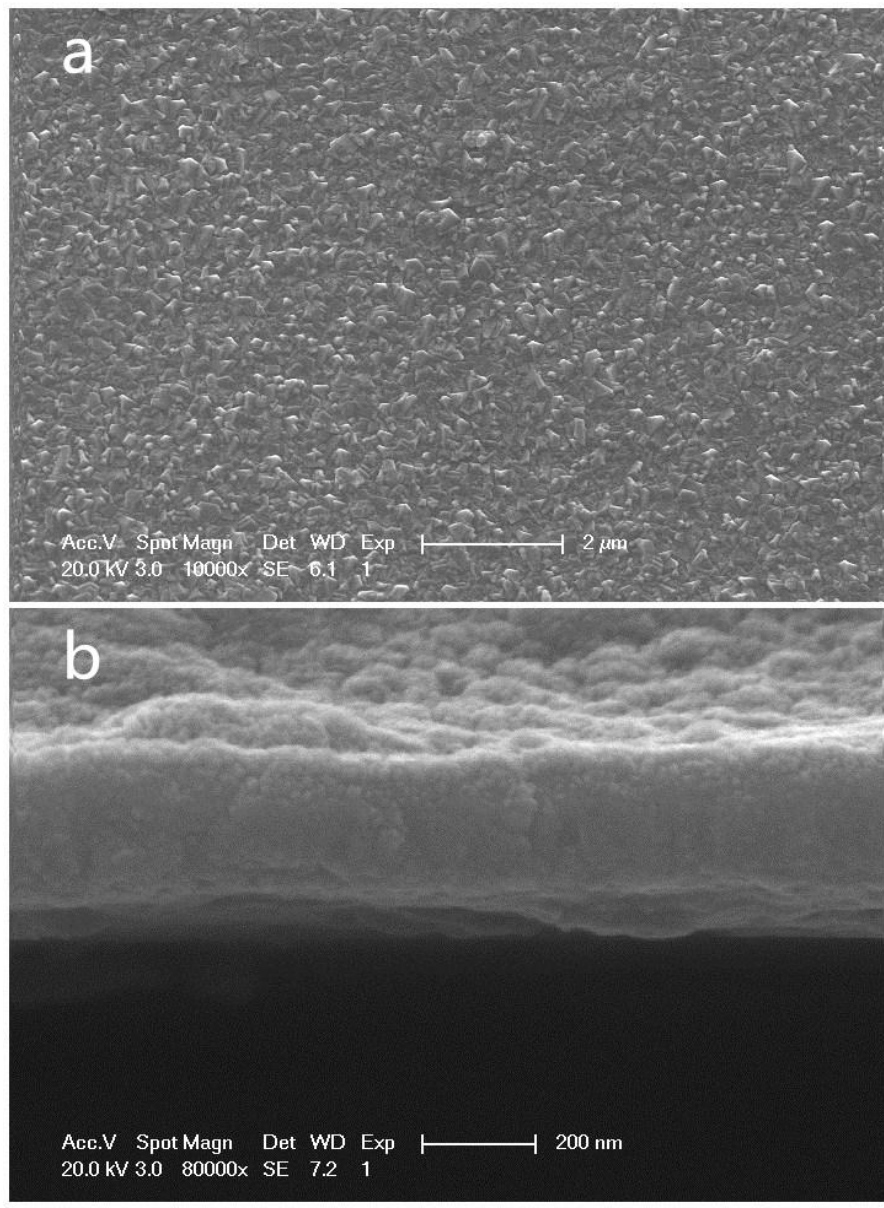

Figure 1. The surface (a) and cross section (b) SEM images of the $\mathrm{Au} / \mathrm{CZT}$ sample after annealing.

To further study the photo response of Au/CZT detectors after annealing, the photocurrent variation with the intensity of X-ray illumination are shown in Figure 3. Since the X-ray intensity is linearly proportional to the electron current that bombards the $\mathrm{Cu}$ target, the bombardment current is used to implicate the photon number of X-ray. As observed in Figure 3-a, the photo current is increased with the $\mathrm{X}$-ray intensity. An inflection point can be obviously seen around 10-30 V for all curves. Below the inflection points, the photon current is increased linearly with applied voltage, suggesting that the number of the photo generated carriers is collected based on applied electron field. At the inflection point, all photo generated carriers are collected. After the inflection point, the current is still increased because of the illumination-related impact 
ionization effect. It should be noted that the inflection point is increased from about $10 \mathrm{~V}$ to $30 \mathrm{~V}$ with increasing the intensity of X-ray and then the photo generated carriers. To further prove the impact ionization effect after the inflection point, the variation of photo current at $50 \mathrm{~V}$ and $200 \mathrm{~V}$ with the intensity of X-ray is given in Figure 3-b. Both curves are linearly increased with the intensity of $\mathrm{X}$ ray. However, it can be clearly seen that the incept of curve for $200 \mathrm{~V}$ at $0 \mathrm{~V}$ is around $4.72 \times 10^{-6} \mathrm{~mA}$, while that for $50 \mathrm{~V}$ is around $4.90 \times 10^{-7} \mathrm{~mA}$. This strongly implies that the impact ionization happens at the electric field above the inflection point.
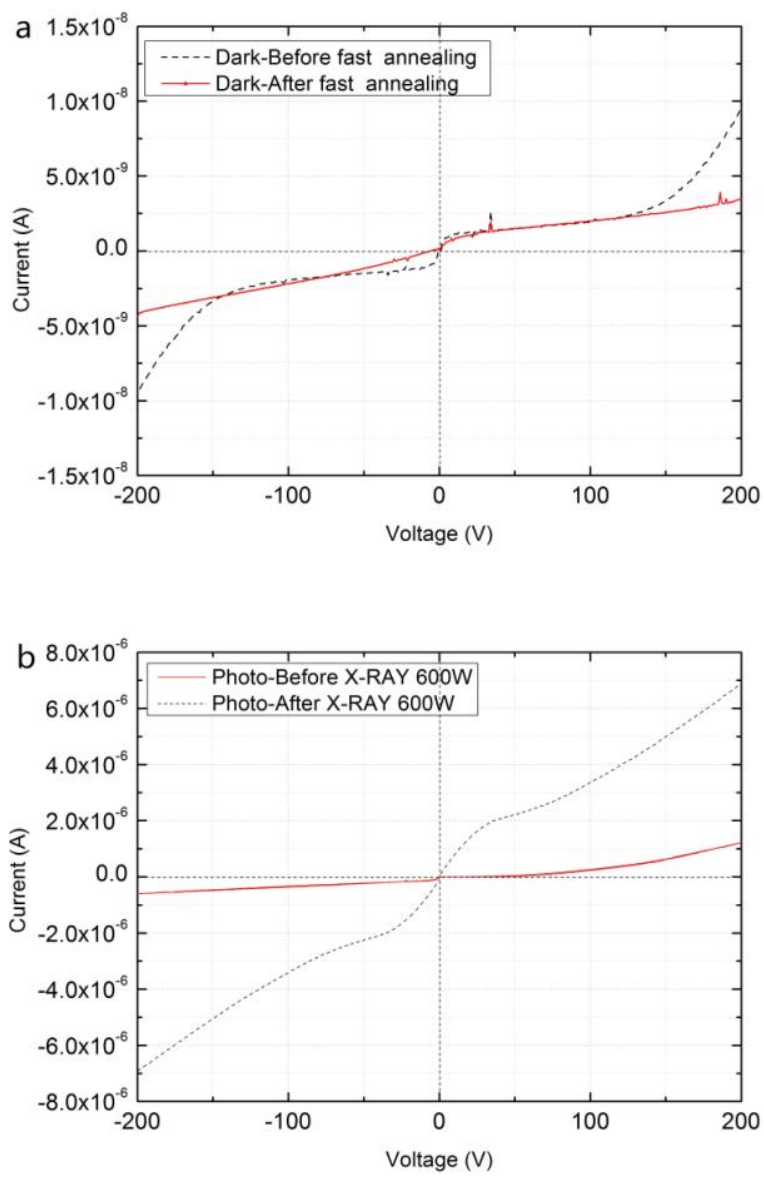

Figure 2. Dark I-V (a) and photocurrent responses (b) of detector before and after annealing.

The radiation damage is a key factor for high energy particle detector. The radiation damage of $\mathrm{Au} / \mathrm{CZT}$ detector after annealing is studied by exposing to X-ray for $0.5 \mathrm{~h}$. It can be observed from Figure 4 that the photo current is very stable below the inflection point, even after the $0.5 \mathrm{~h}$ exposure, while the photo current above the inflection point decreases with exposure time. This decrease seems to come from the passivation of the defect in CZT bulk after illumination. As demonstrated above, the photo current above the inflection point arises from both the photo generated carrier and the illumination-related impact ionization. This impact ionization is strongly dependent on the local electric field and thus the active defects. After a long X-ray exposure, some defect may capture some carriers and then be passivated by those carriers.
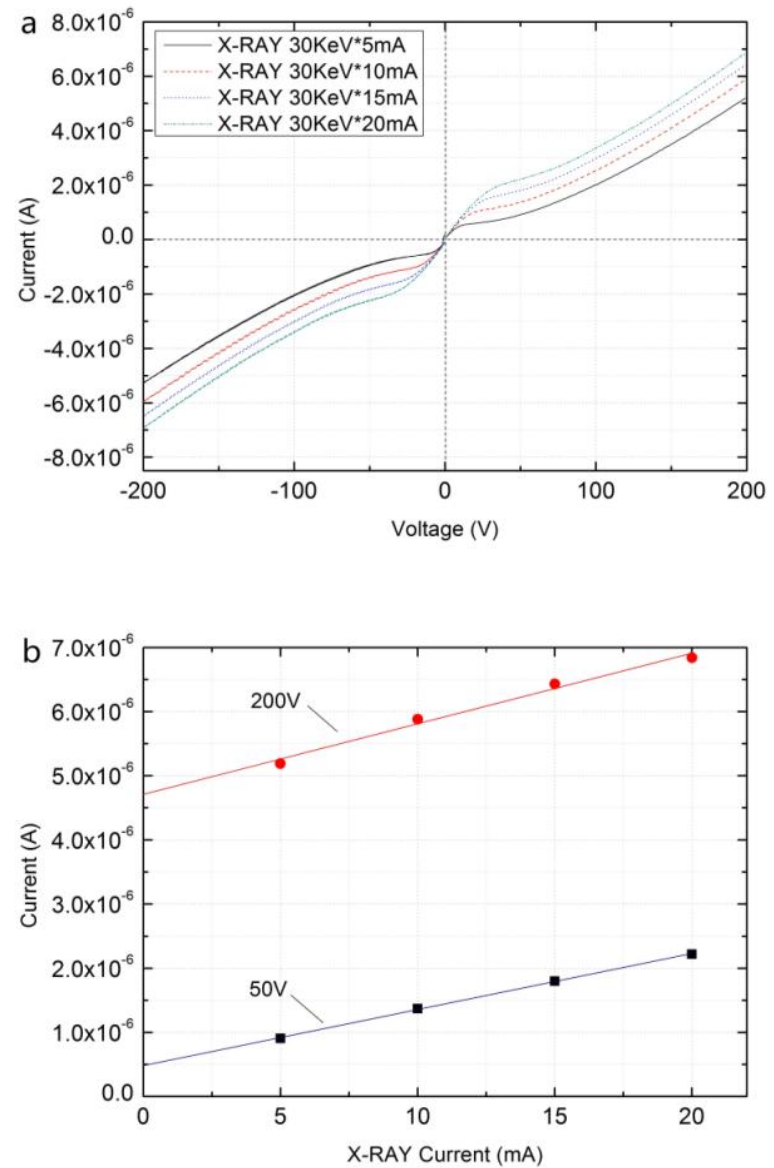

Figure 3. (a) I-V curve of radiation response of CZT detector after annealing; (b) the variation of photo current with the increase of X-ray current.

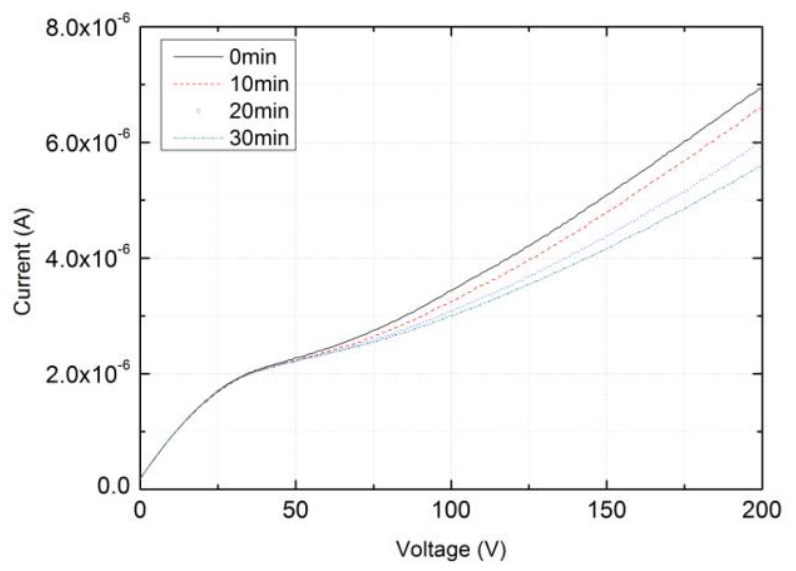

Figure 4. The radiation damage of Au/CZT detector after annealing with exposure to X-ray under $30 \mathrm{KeV}$ and $20 \mathrm{~mA}$ for $0.5 \mathrm{~h}$.

\section{CONCLUSIONS}

After RTA, the linearity of I-V curve is much better than before. Meanwhile, the interface becomes uniform and this leakage channel is eliminated. As observed, photo current increases with the X-ray intensity. It can be obviously seen that there is an 
inflection point around $10-30 \mathrm{~V}$ for all curves. Below the inflection points, with the increase of applied voltage, photon current increases linearly. After the inflection point, the current still increases because of the illumination-related impact ionization effect. In addition, radiation damage would appear when the detector exposed to X-ray under $30 \mathrm{KeV}$ and $20 \mathrm{~mA}$ for $0.5 \mathrm{~h}$. The photon current does not change over time before the inflection point, however, it changes after the inflection point.

\section{ACKNOWLEDGE}

This work was supported by National Natural Science Foundation of China (No.11375112), Science and Technology Commission of Shanghai (No.11530500200) and Innovation Program of Shanghai Municipal Education Commission (No. 12ZZ096).

\section{REFERENCES}

[1] Bell, S.J. 2013. A multi-technique characterization of electroless gold contacts on single crystal CdZnTe radiation detectors, Journal of Physics D: Applied Physics 46(45): 455502.

[2] Bensalah,H. 2011. The effect of etching time on the CdZnTe surface, Applied Surface Science 257(10): 4633.

[3] Crocco,J. 2011. Study of the effects of edge morphology on detector performance by leakage current and cathodoluminescence, Nuclear Science, IEEE Transactions on 58(4): 1935.

[4] Duff, M. C. 2008. Effect of surface preparation technique on the radiation detector performance of CdZnTe, Applied Surface Science, 254(9): 2889.

[5] Li, Q. 2006. Metal-CdZnTe contact and its annealing behaviors, Applied Surface Science 253(3): 1190.

[6] Morton, E. 2001. Investigation of Au-CdZnTe contacts using photovoltaic measurement, Nuclear Instruments and Methods in Physics Research Section A: Accelerators, Spectrometers, Detectors and Associated Equipment 458(1): 558.

[7] Park, S. H. 2008. Surface Passivation Effect on CZTMetal Contact, Nuclear Science, IEEE Transactions on 55(3): 1547.

[8] Schlesinger, T.E. 2001. Cadmium zinc telluride and its use as a nuclear radiation detector material, Materials Science and Engineering 32(4): 103.

[9] Tari, S. 2014. Structural and electronic properties of gold contacts on CdZnTe with different surface finishes for radiation detector applications, Journa of Electronic Materials 43(8): 2980.

[10] Zheng,Q. 2011a. Electroless plating of $\mathrm{Au}, \mathrm{Pt}$, or $\mathrm{Ru}$ thin film layer on CdZnTe, Nuclear Science Symposium and Medical Imaging Conference, 2011 IEEE: 4848.

[11] Zheng,Q. 2011b. Comparison of radiation detector performance for different metal contacts on $\mathrm{CdZnTe}$ deposited by electroless deposition method, Crystal Research and Technology 46(11): 1131.

[12] Zheng,Q. 2012. Electroless deposition of $\mathrm{Au}, \mathrm{Pt}$, or $\mathrm{Ru}$ metallic layers on CdZnTe, Thin Solid Films, 525: 56. 